\title{
Disfunção temporomandibular e fatores psicológicos: uma revisão de literatura
}

\author{
Temporomandibular dysfunction association with psychological factors: a literature review \\ Disfunción temporomandibular y factores psicológicos: revisión de la literatura
}

Recebido: 19/02/2021 | Revisado: 03/03/2021 | Aceito: 03/03/2021 | Publicado: 10/03/2021

Italo Hudson Tavares Maia

ORCID: https://orcid.org/0000-0003-4274-115X
Faculdade Paulo Picanç, Brasil

E-mail: italohudson98@hotmail.com

Tainah Oliveira Rifane

ORCID: https://orcid.org/0000-0001-6120-1672

Faculdade Paulo Picanço, Brasil

E-mail: tainah08@gmail.com

Andressa Silva de Oliveira

ORCID: https://orcid.org/0000-0001-9015-844X

Faculdade Paulo Picanço, Brasil

E-mail: andressa_oliveira21@outlook.com

Francisbênia Alves Silvestre

ORCID: https://orcid.org/0000-0001-8620-8678

Faculdade Paulo Picanço, Brasil

E-mail: beniaasilvestre@gmail.com

Bárbara de Fátima Barboza de Freitas

ORCID: https://orcid.org/0000-0002-7127-8056 Faculdade Paulo Picanço, Brasil

E-mail: barbarafreitas130599@gmail.com

Aline Kércia Adeodato Leitão

ORCID: https://orcid.org/0000-0001-6338-9939

Faculdade Paulo Picanço, Brasil

E-mail: aline.kercia@facpp.edu.br

Victor Pinheiro Feitosa

ORCID: https://orcid.org/0000-0001-8795-9967 Faculdade Paulo Picanço, Brasil

E-mail: victor.feitosa @ facpp.edu.br

Eveline Guedes Fernandes

ORCID: https://orcid.org/0000-0003-1723-5052 Faculdade Paulo Picanço, Brasil

E-mail: eveline_guedes@yahoo.com.br

Diego Martins de Paula

ORCID: https://orcid.org/0000-0003-0218-9436

Faculdade Paulo Picanço, Brasil

E-mail: diego.martins @ facpp.edu.br

\begin{abstract}
Resumo
O estudo objetivou revisar a literatura sobre a relação entre disfunção temporomandibular (DTM) e fatores psicológicos. Trata-se de uma revisão de literatura com abordagem qualitativa. As bases de dados foram PubMed e BIREME, no período de 2008 a 2018. Como critérios de inclusão: artigos publicados na língua inglesa, com os descritores "temporomandibular joint disorder", "anxiety", "depression", "stress" e "psychological". Foram excluídos os artigos de revisão de literatura e artigos onde o componente psicológico foi apenas citado e não avaliado propriamente. Caracterizaram-se oito artigos, dos quais os resultados apontaram a correlação significativa entre DTM e comorbidades psicossociais, somáticas e comportamentais. Os fatores psicológicos como somatização, depressão, ansiedade e angústia, principalmente relacionadas ao gênero feminino, configuram um impacto significativo na prevalência de DTM. Nota-se que existe relação aos fatores citados, dessa forma, necessita da assistência interdisciplinar em virtude da natureza físico-psicológica, ou seja, participação da psicologia para intensificação do tratamento odontológico.
\end{abstract}

Palavras-chave: Disfunção temporomandibular; Ansiedade; Depressão.

\section{Abstract}

Therefore, this study aimed to review the literature focusing on the relationship between TMD and psychological factors. This is a literature review with a qualitative approach. The databases were PubMed and Bireme, from 2008 to 2018. As inclusion criteria: articles published in the English language, with the descriptors "temporomandibular Joint disorder", "anxiety", "depression", "stress" and "psychological". Articles of literature review and articles where the 
psychological component was only cited and not evaluated were excluded. Eight articles were characterized, of which the results pointed out the significant correlation between psychosocial, somatic and behaviour comorbidities. Psychological factors such as somatization, depression, anxiety and distress, mainly related to the female gender, have a significant impact on the prevalence of TMD. It is noted that there is a relation to the cites factors. In this regard, it requires the interdisciplinary assistance due to the physical-psychological nature, in other words, participation of psychology to intensify the dental treatment.

Keywords: Temporomandibular dysfunction; Anxiety; Depression.

\section{Resumen}

El estudio tuvo como objetivo revisar la literatura sobre la relación entre el trastorno temporomandibular (TTM) y factores psicológicos. Se trata de una revisión de la literatura con enfoque cualitativo. Las bases de datos fueron PubMed y BIREME, de 2008 a 2018. Como criterios de inclusión: artículos publicados en inglés, con los descriptores "trastorno de la articulación temporomandibular", "ansiedad", "depresión”, "estrés" y "psicológico". Se excluyeron los artículos de revisión de la literatura y aquellos en los que el componente psicológico solo se citó y no se evaluó adecuadamente. Se caracterizaron ocho artículos, de los cuales los resultados mostraron una correlación significativa entre TMD y comorbilidades psicosociales, somáticas y conductuales. Factores psicológicos como somatización, depresión, ansiedad y angustia, principalmente relacionados con el género femenino, configuran un impacto significativo en la prevalencia de TTM. Se observa que existe una relación con los factores mencionados, por lo que requiere asistencia interdisciplinaria debido al carácter físico-psicológico, es decir, la participación de la psicología para intensificar el tratamiento odontológico.

Palabras clave: Trastorno temporomandibular; Ansiedad; Depresión.

\section{Introdução}

Disfunção Temporomandibular (DTM) é uma desordem que envolve os músculos mastigatórios, articulação temporomandibular (ATM) e estruturas associadas. Sua etiologia é multifatorial e envolve diferentes aspectos tais como problemas musculares, oclusais, psicológicos, anormalidades estruturais e posturais, hábitos parafuncionais, processos degenerativos e lesões traumáticas (Maydana et al., 2010). Estudos mostram que 50 a $70 \%$ da população mundial têm pelo menos um sinal e sintoma de DTM em algum estágio da vida incluindo dor, limitação do movimento mandibular e barulho articular (Conti et al., 2012).

Os fatores de origem psicológica como depressão, somatização e ansiedade tem um importante papel na etiologia e evolução sintomatológica de DTM, contribuindo para o aparecimento ou perpetuação da desordem por meio do aumento da atividade muscular e tensão dos músculos da face (Ferreira et al., 2009; Licini et al., 2009). A dor crônica originada pela DTM pode estar relacionada ao estado psicológico, apresentando grande influência no comportamento social e gerando um grande impacto na qualidade de vida (Zavanelli et al., 2017). Pacientes com severa depressão causada por dor crônica podem ter dificuldade de realizar atividades diárias como trabalhar e estudar, enquanto pacientes que relatam alta intensidade de dor relacionada à DTM podem apresentar altos níveis de somatização (Su et al., 2017).

O risco para desenvolvimento de ansiedade e depressão em pacientes com DTM é maior em mulheres, indivíduos com suporte social deficiente e pacientes com dor miofascial. Reconhecer pacientes com risco de problemas psicossociais é importante por ser praticamente impossível requerer avaliação psiquiátrica para todos os pacientes com DTM e o tratamento desses indivíduos usando apenas técnicas biomecânicas clássicas é geralmente insuficiente (Pinto et al., 2017). Sendo assim é muito importante o diagnóstico precoce e uma abordagem multiprofissional, onde o papel do psicólogo é fundamental no diagnóstico correto e acompanhamento para o sucesso do tratamento desses pacientes (Su et al., 2017).

Dessa forma, o objetivo do presente estudo é apresentar uma revisão de literatura para avaliar a relação entre problemas psicológicos como ansiedade, stress, depressão, somatização e disfunção temporomandibular. 


\section{Metodologia}

O presente trabalho trata-se de um estudo de natureza qualitativa (Pereira et al., 2018), dessa forma, foi elaborado a pesquisa foi realizada nas bases de dados PubMed e BIREME, buscando estudos realizados em humanos publicados em inglês entre as datas de 03/05/2013 e 03/05/2018 com os descritores: "Temporomandibular joint disorder", "anxiety", "depression", "stress", "psychological". Sendo também incluída pesquisa manual da lista de referência de artigos selecionados.

\subsection{Critérios de elegibilidade}

\subsubsection{Critérios de inclusão}

- Artigos na língua inglesa.

- Estudos feitos em humanos.

- Artigos que relacionam o componente psicológico com disfunção temporomandibular.

\subsubsection{Critérios de exclusão:}

- Artigos de revisão de literatura.

- Artigos onde o componente psicológico foi apenas citado e não avaliado.

\subsection{Pesquisa}

Tabela 1. Resumo da busca no Bireme.

\begin{tabular}{|c|c|}
\hline Descritores & Números de artigos achados \\
\hline "temporomandibular joint disorder"+ "anxiety" & 299 \\
\hline $\begin{array}{l}\text { "temporomandibular joint disorder"+ “anxiety”+ } \\
\text { "depression" }\end{array}$ & 150 \\
\hline $\begin{array}{l}\text { "temporomandibular joint disorder" +“anxiety"+ } \\
\text { "depression"+ "stress" }\end{array}$ & 54 \\
\hline $\begin{array}{l}\text { "temporomandibular joint disorder"+ "anxiety"+ } \\
\text { "depression"+ "stress"+ "psychological" }\end{array}$ & 49 \\
\hline
\end{tabular}

Fonte: Autores. 
Tabela 2. Resumo encontrado no PubMed.

\begin{tabular}{|c|c|}
\hline Descritores & Números De Artigos Achados \\
\hline "temporomandibular joint disorder"+ "anxiety" & 328 \\
\hline $\begin{array}{c}\text { "temporomandibular joint disorder"+ "anxiety"+ } \\
\text { "depression" }\end{array}$ & 166 \\
\hline $\begin{array}{c}\text { "temporomandibular joint disorder" +“anxiety"+ } \\
\text { "depression"+ "stress" }\end{array}$ & 55 \\
\hline $\begin{array}{l}\text { "temporomandibular joint disorder"+ "anxiety"+ } \\
\text { "depression"+ "stress"+ "psychological” }\end{array}$ & 49 \\
\hline
\end{tabular}

Fonte: Autores.

\subsection{Processo de triagem dos artigos}

Inicialmente os artigos foram selecionados por título e resumo de acordo com a estratégia de pesquisa descrita artigos que apareceram em mais de uma base de dados foram considerados somente uma vez. Os títulos foram analisados por dois revisores (I.H.M., A.K.L.), independentemente, os que eram interessantes para essa pesquisa tiveram os resumos lidos, ou se o resumo era insuficiente o artigo completo foi lido. Em caso de discordância, um terceiro autor foi contatado.

Tabela 3. Resultado da busca dos artigos.

\begin{tabular}{cc}
\hline & Número de artigos \\
\hline Encontrados no PUBMED & 598 \\
Encontrados no BIREME & 552 \\
Total de artigos encontrados & 1150 \\
Excluindo-se a duplicidade & 650 \\
Excluídos após a leitura do título & 630 \\
Excluídos após a leitura dos resumos & 13 \\
Artigos selecionados & 07 \\
\hline
\end{tabular}

Fonte: Autores. 


\section{Resultados e Discussão}

Para análise dos resultados dessa revisão, a Tabela 4 foi confeccionada contendo: o número de participantes, a faixa etária, os métodos de avaliação, os fatores psicossociais e os principais achados. Os fatores mais encontrados foram distúrbios do sono e ansiedade.

Tabela 4. Resultados metodológicos:

\begin{tabular}{|c|c|c|c|c|c|}
\hline Artigo & $\begin{array}{l}\text { Número de } \\
\text { participantes }\end{array}$ & $\begin{array}{l}\text { Faixa } \\
\text { etária }\end{array}$ & Métodos de avaliação & $\begin{array}{c}\text { Fatores } \\
\text { psicossociais } \\
\text { analisados }\end{array}$ & Principais achados \\
\hline $\begin{array}{c}\text { Pesqueira et al., } \\
2010\end{array}$ & 150 & $\begin{array}{l}17-30 \\
\text { anos }\end{array}$ & $\begin{array}{c}\text { Foram analisados por RDC/TMD e } \\
\text { questionários de ansiedade. }\end{array}$ & Ansiedade & $\begin{array}{l}\text { Associação positiva entre } \\
\text { ansiedade e DTM. }\end{array}$ \\
\hline $\begin{array}{c}\text { Lajnert et al., } \\
2010\end{array}$ & 90 & $\begin{array}{l}22-67 \\
\text { anos }\end{array}$ & $\begin{array}{c}\text { Feitas por testes como RDC/TMD e } \\
\text { testes psicológicos }\end{array}$ & $\begin{array}{l}\text { Ansiedades, } \\
\text { depressão e } \\
\text { somatização }\end{array}$ & $\begin{array}{l}\text { Altos níveis de ansiedades, } \\
\text { depressão e somatização em } \\
\text { pacientes de DTM em relação } \\
\text { aos pacientes saudáveis. }\end{array}$ \\
\hline $\begin{array}{l}\text { Lei et al., } \\
2015\end{array}$ & 510 & $\begin{array}{l}11-79 \\
\text { anos }\end{array}$ & $\begin{array}{c}\text { RDC/TMD e escala de auto avaliação } \\
\text { de sono (SRSS) }\end{array}$ & $\begin{array}{l}\text { Distúrbios de sono e } \\
\text { angústia }\end{array}$ & $\begin{array}{c}\text { O distúrbio de sono e os } \\
\text { sintomas de angústia são } \\
\text { possíveis indicadores de risco } \\
\text { para a dor miofascial. }\end{array}$ \\
\hline $\begin{array}{l}\text { Lei et al., } \\
2016\end{array}$ & 578 & $\begin{array}{l}12-18 \\
\text { anos }\end{array}$ & $\begin{array}{l}\text { Para avaliar a qualidade do sono e os } \\
\text { estresses psicológicos foi utilizado a } \\
\text { versão chinesa de Pittsburgh Sleep } \\
\text { Quality Index (CPSQI) e Stress Scales- } \\
21 \text { (DASS-21), além do RDC/TMD }\end{array}$ & $\begin{array}{l}\text { Distúrbios de sono e } \\
\text { estresse psicológicos }\end{array}$ & $\begin{array}{l}\text { Foram avaliados a presença de } \\
\text { DTM em adolescentes com } \\
\text { Distúrbios de sono e estresse } \\
\text { psicológicos }\end{array}$ \\
\hline $\begin{array}{c}\text { Al-Khotani et } \\
\text { al., } \\
2016\end{array}$ & 456 & $\begin{array}{l}10-18 \\
\text { anos }\end{array}$ & $\begin{array}{l}\text { Com os testes clínicos de dor e versão } \\
\text { árabe da escala de auto relatório }\end{array}$ & $\begin{array}{l}\text { Psicossociais, } \\
\text { Somáticos e } \\
\text { comportamentais }\end{array}$ & $\begin{array}{l}\text { Pode-se verificar que existem } \\
\text { associações significativas } \\
\text { entre comorbidades e DTM } \\
\text { em crianças e adolescente }\end{array}$ \\
\hline $\begin{array}{l}\text { Su et al., } \\
2017\end{array}$ & 320 & $* *$ & Questionários de estresse e sintomas & $\begin{array}{c}\text { Somatização e } \\
\text { depressão }\end{array}$ & $\begin{array}{l}\text { A somatização foi o melhor } \\
\text { preditor de intensidade da dor } \\
\text { e a depressão foi pior preditor } \\
\text { de deficiência de dor }\end{array}$ \\
\hline $\begin{array}{c}\text { Drysdale et } \\
\text { al., } \\
2017\end{array}$ & 469 & $* *$ & $\begin{array}{c}\text { Avaliação da história médica através de } \\
\text { prontuários }\end{array}$ & $* *$ & $\begin{array}{c}\text { Medicamentos específicos do } \\
\text { sistema nervoso central são } \\
\text { associados a sintomas de } \\
\text { DTM }\end{array}$ \\
\hline
\end{tabular}

(*) informação não fornecida pelo o artigo analisado

(**) informação cedida encontrasse incompleta

RDC/TMD-Research Diagnostic Criteria for Temporomandibular Disorders, Critérios diagnósticos para pesquisa em DTM.

Fonte: Autores. 
Dessa forma, os resultados apontam a correlação significativa entre DTM e comorbidades psicossociais, somáticas e comportamentais. Esses fatores juntos da depressão, ansiedade e angústia, principalmente relacionados ao gênero feminino, possuem um impacto significativo na prevalência de DTM.

A relação do gênero feminino teve impacto significativo da prevalência de DTM, e é algo que também foi levado em consideração. Os resultados em relação aos gêneros foram detalhados na Tabela 5.

Tabela 5. Relação dos gêneros na prevalência de DTM.

\begin{tabular}{|c|c|c|c|c|c|}
\hline Autor & Masculino & Feminino & Ambos & $\begin{array}{c}\text { Não foi } \\
\text { avaliado }\end{array}$ & Gênero mais avaliado nos artigos \\
\hline $\begin{array}{l}\text { Pesqueira et al., } \\
2010\end{array}$ & & & & $\mathrm{x}$ & $*$ \\
\hline $\begin{array}{l}\text { Lajnert et al., } \\
2010\end{array}$ & & $\mathrm{x}$ & & & $\begin{array}{c}\text { Feminino } \\
(100 \%)\end{array}$ \\
\hline $\begin{array}{l}\text { Lei et al., } \\
2015\end{array}$ & & & $\mathrm{x}$ & & $\begin{array}{c}\text { Feminino } \\
(75 \%)\end{array}$ \\
\hline $\begin{array}{l}\text { Lei et al., } \\
2016\end{array}$ & & & $\mathrm{x}$ & & $\begin{array}{c}\text { Feminino } \\
(64,2 \%)\end{array}$ \\
\hline $\begin{array}{l}\text { Al-Khotani et al., } \\
2016\end{array}$ & & & $x$ & & $\begin{array}{c}\text { Feminino } \\
(64,3 \%)\end{array}$ \\
\hline $\begin{array}{l}\text { Diracoglu et al., } \\
2016\end{array}$ & & & $\mathrm{x}$ & & $\begin{array}{c}\text { Feminino } \\
(84 \%)\end{array}$ \\
\hline $\begin{array}{l}\text { Su et al., } \\
2017\end{array}$ & & & $\mathrm{x}$ & & $\begin{array}{c}\text { Feminino } \\
(78,2 \%)\end{array}$ \\
\hline
\end{tabular}

(*) informação não fornecida pelo o artigo analisado Fonte: Autores.

A porcentagem informada na Tabela 2 é em relação de quantos por centos do grupo total de pacientes analisados pertencia ao gênero citado. Por conseguinte, podemos observar que a porcentagem no gênero feminino foi significante maior do que os do sexo masculino. Logo, fatores psicológicos em mulheres foram mais relatados e citados na literatura, podendo comprovar que o impacto de DTM em pacientes do gênero feminino é mais prevalente.

A etiologia da DTM é multifatorial podendo ser por traumas, fatores anatômicos, patofisiológicos e psicológicos, atuando sozinhos ou em combinação. Nos artigos revisados foram encontradas associações de DTM com depressão, ansiedade, estresse e distúrbios do sono nas mais diferentes amostras que englobam diversas faixas etárias.

No presente estudo, os artigos selecionados foram utilizados como medidas de avaliação para disfunção temporomandibular os critérios do RDC/TMD e para avaliar os sintomas dos fatores psicológicos foram utilizados os devidos testes psicológicos. De acordo com os estudos de Pesqueira et al, (2010); Lajnert et al, (2010), sinais e sintomas de ansiedade foram avaliados, e observou-se uma associação efetiva com a DTM. Outros fatores também foram analisados como: depressão e somatização e houve também uma correlação positiva com a disfunção. 
A depressão atualmente tem um grande impacto epidemiológico, com sintomas diversos. Sua correlação com a DTM foi avaliada no presente estudo. Su et al, (2017) enfatiza isso, mostrando que há um melhor preditor para intensidade de dor sendo a somatização e a depressão como um pior preditor para falta de dor em pacientes com sinais de DTM.

Outro fator que também foi citado e analisado foi o distúrbio do sono e estresse psicológico. Logo, tais fatores podem ocorrer em um mesmo sujeito simultaneamente ou em sequência, sendo consideradas condições de comorbidades que são possíveis indicadores de risco para DTM (Lei et al., 2015 e Selaimen et al., 2006).

Segundo Lei et al, (2016), adolescentes que relatam sintomas de DTM tem mais severo estrese psicológico e distúrbio do sono que adolescentes sem sintomas. Cerca de $90 \%$ das pessoas que possuem desordem temporomandibular relatam uma baixa qualidade no sono e ao serem analisados os exames de polissonografia (PSG) de pacientes com DTM são observados comumente distúrbios como bruxismo do sono e apneia obstrutiva do sono (Rener-Sitara et al., 2016).

Al-Khotani et al, (2016) relatou que em adolescentes e crianças existe uma relação significativa de comorbidades e DTM, evidenciando que foi dito anteriormente. Estudos têm mostrado que pacientes com DTM tem altos níveis de estresse psicológico e afetivo e mostram a importância de fatores psicológicos em predispor, precipitar e perpetuar a DTM (Lei et al., 2015; Kindler et al., 2012; Smith et al., 2009).

Outra circunstância que também foi explorada foi a maior predominância do gênero feminino entre os pacientes que relatam algum tipo de sinais ou sintomas das DTM. Alguns artigos sugerem que há uma possível ligação entre a patogenicidade da DTM e o hormônio sexual estrogênio que as mulheres possuem, principalmente, durante o período menstrual devido, as altas taxas de hormônios liberado. Embora também deva ser contextualizado o nexo entre a DTM e o mecanismo de modulação de dor, uma vez que as mulheres apresentam uma maior sensibilidade para a maioria das modalidades de dor (Ferreira et al., 2016).

Além dos fatores fisiológicos deve ser levado em consideração que as mulheres ainda são o público que mais buscam tratamento para essas desordens enquanto os homens quase não vão à busca do tratamento dessa forma, podendo ter um menor índice de presença quando são levantados em dados para as pesquisas desse assunto. Salientando-se, da necessidade de estudos mais aprofundados neste campo, pois a literatura ainda não tem uma hipótese bem resolvida sobre o assunto abordado.

Ademais, situações no qual o paciente tem bruxismo do sono (BS) podem estar associadas ao uso de fármacos como: duloxetina, paroxetina e venlafaxina entre adultos, enquanto fármacos barbitúricos e metilfenidato podem exibir uma associação positiva com a presença de BS entre as populações mais jovens (Melo et al., 2018).

Drysdale et al, (2017) foi analisado a relação entre os fármacos que regulam doenças como depressão e déficit de atenção e as DTM. Não havendo assim diferença significativa entre os grupos que foram prescritos com medicamentos para TDAH e os que não foram, mas sim uma diferença entre os medicamentos ministrados. Entretanto, pacientes que tem uma predisposição à DTM podem se beneficiar com a ingestão de tais medicamentos

\section{Conclusão}

De acordo com os resultados concluímos que há correlação positiva entre desordens psicológicas citadas e DTM, sendo que uma desordem pode predispor o surgimento da outra, principalmente no gênero feminino, e que existe necessidade da intensificação do trabalhado do psicólogo para assistir a interdisciplinaridade do trabalho do cirurgião-dentista em virtude da natureza físico-psicológica da disfunção temporomandibular. Assim, são necessários mais estudos clínicos futuros que fortaleça essa correlação, visto que o assunto é bastante atual em nossa sociedade. 


\section{Referências}

Adriana, S. P., Dorlivete, M. S., Fabio, J. P., \& Ricardo, S. (2018). Metodologia da pesquisa científica. UFSM. .https://repositorio.ufsm.br/bitstream/handle/1/15824/Lic_Computacao_Metodologia-Pesquisa-Cientifica.pdf?sequence=1

Al-Khotani, A., Naimi-Akbar, A., Gjelset, M., Albadawi, E., Bello, L., Hedenberg-Magnusson, B., \& Christidis, N. (2016). The associations between psychosocial aspects and TMD-pain related aspects in children and adolescents. J Headache Pain, 17(1), 30.

Conti, P. C. R., Pinto-Fiamengui, L. M. S., Cunha, C. O., \& Conti, A. C. C. F. (2012). Dor orofacial e disfunção temporomandibular: o impacto na saú de bucal e na qualidade de vida. Brazilian Oral Research, 26 (spe1), 120-123.

Drysdale, A. T., Grosenick, L., Downar, J., Dunlop, K., Mansouri, F., Meng, Y., et al. (2017). Resting-state connectivity biomarkers define neurophysiological subtypes of depression. Nature medicine, 23(1), 28-38.

Ferreira, C. L. P., Silva, M. A. M. R., \& Felício, C. M. (2016). Signs and symptoms of temporomandibular disorders in women and men. CoDAS, 28(1), 1721.

Ferreira, K. D. M., Guimarães, J. P. Batista, C. H. T., et al. (2009). Fatores psicológicos relacionados à sintomatologia crônica das desordens temporomandibulares - revisão de literatura. RFO UPF, 14(3), 262-7.

Kindler, S., Samietz, S., Houshmand, M., Grabe, H. J., Bernhardt, O., Biffar, R., Kindler, S., Samietz, S., Houshmand, M., Grabe, H. J., Bernhardt, O., Biffar, R., et al. (2012). Depressive and anxiety symptoms as risk factors for temporomandibular joint pain: a prospective cohort study in the population. $J$ Pain, $13(12), 1188-97$.

Lajnert, V., Francisković, T., Grzic, R., Pavicić, D. K., Bakarbić, D., Buković, D., Celebić, A., Braut, V., \& Fugosić, V. (2010). Depression, somatization and anxiety in female patients with temporomandibular disorders (TMD). Coll Antropol, 4(4), 1415-9.

Lei, J., Fu, J., Yap, A. U., \& Fu, K. Y. (2016). Temporomandibular disorders symptoms in Asian adolescents and their association with sleep quality and psychological distress. Cranio, 34(4), 242-9.

Lei, J., Liu, M. Q., Yap, A. U., \& Fu, K. Y. (2015). Sleep disturbance and psychologic distress: prevalence and risk indicators for temporomandibular disorders in a Chinese population. J Oral Facial Pain Headache, 29(1), 24-30.

Licini, F., Nojelli, A., Segù, M., \& Collesano, V. (2009). Role of psychosocial factors in the etiology of temporomandibular disorders: relevance of a biaxial diagnosis 2020. Minerva Stomatologica, 58(11-12), 557-66.

Maydana, A. V., Tesch, R. de S., Denardin, O. V. P., Ursi, W. J. S., \& Dworkin, S. F. (2010). Possíveis fatores etiológicos para desordens temporomandibulares de origem articular com implicações para diagnóstico e tratamento. Dental Press Journal of Orthodontics, 15(3), 78-86. https://doi.org/10.1590/S2176-94512010000300010

Melo, G., Dutra, K. L., Rodrigues, F. R., Ortega, A. O. L., Porporatti, A. L., et al. (2018). Association between psychotropic medications and presence of sleep bruxism: a systematic review. J Oral Rehabil, 45, 545-554.

Pesqueira, A. A., Zuim, P. R. J., Monteiro, D. R., \& Garcia, A. R. (2010). Relationship between psychological factors and symptoms of TMD in university undergraduate students. Acta odontológica latino-americana. AOL, 23(3), 182-187.

Pinto, S. G. R., Leite, A. M. W., Sampaio, S. L., \& Sanchez, O. M. (2017). Associação entre sinais e sintomas de disfunção temporomandibular com depressão em universitários: estudo descritivo. Revista Dor, 18(3).

Rener-Sitar, K., John, M. T., Pusalavidyasagar, S. S., Bandyopadhyay, D., \& Schiffman, E. L. (2016). Sleep quality in temporomandibular disorder cases. Sleep medicine, 25, 105-112.

Selaimen, C. M., Jeronymo, J. C., Brilhante, D. P., \& Grossi, M. L. (2006). Sleep and depression as risk indicators for temporomandibular disorders in a crosscultural perspective: a case-control study. Int J Prosthodont, 19(2), 154-61.

Smith, M. T., Wickwire, E. M., Grace, E. G., Edwards, R. R., Buenaver, L. F., Peterson, S., Klick, B., \& Haythornthwaite, J. A. (2009). Sleep disorders and their association with laboratory pain sensitivity in temporomandibular joint disorder. Sleep, 32(6), 779-90.

Su, N, Lobbezoo, F., van Wijk, A., van der Heijden, G. J., \& Visscher, C. M. (2017). Associations of pain intensity and pain related disability with psychological and sociodemographic factors in patients with temporomandibular disorders: cross-sectional study at a specialised dental clinic. $J$ Oral Rehabil, 44(3), 187-96.

Zavanelli, A. C., Rezende, M. C. R. A., Santos, O. M., \& Neto, R. S. (2017). Integration of Psychology and Dentistry in TMD: a systematized review. Arch Health Invest, 6(11), 530-534. 